\title{
On Bounded Strictly Positive Operators of Closed Range and Some Applications to Asymptotic Hyperstability of Dynamic Systems
}

\author{
M. De la Sen \\ Institute of Research and Development of Processes, University of the Basque Country, Campus of Leioa (Bizkaia), \\ Apartado 644, 48080 Bilbao, Spain \\ Correspondence should be addressed to M. De la Sen; manuel.delasen@ehu.es
}

Received 7 May 2013; Accepted 27 May 2013

Academic Editor: Luca Guerrini

Copyright (c) 2013 M. De la Sen. This is an open access article distributed under the Creative Commons Attribution License, which permits unrestricted use, distribution, and reproduction in any medium, provided the original work is properly cited.

\begin{abstract}
The problem discussed is the stability of two input-output feedforward and feedback relations, under an integral-type constraint defining an admissible class of feedback controllers. Sufficiency-type conditions are given for the positive, bounded and of closed range feed-forward operator to be strictly positive and then boundedly invertible, with its existing inverse being also a strictly positive operator. The general formalism is first established and the linked to properties of some typical contractive and pseudocontractive mappings while some real-world applications and links of the above formalism to asymptotic hyperstability of dynamic systems are discussed later on.
\end{abstract}

\section{Introduction}

The properties of absolute stability and hyperstability and asymptotic hyperstability of dynamic systems are very important tools in dynamic systems since they are associated with the positivity and boundedness of the energy for all feedback controllers within a wide class characterized by a Popovtype integral inequality, then implying global Lyapunov's stability $[1-8]$. The fact that such properties hold for a class of controllers defined by the Popov inequality, rather than for just some individual one, makes the related theory to be very useful against potential parametrical dispersion of components. The main objective of this paper is the investigation of the strict positivity and stability of bounded positive oneto-one operators with closed range on Hilbert spaces linked to contractive, pseudocontractive, asymptotically pseudocontractive, and asymptotically pseudocontractive in the intermediate sense mappings. See [9-21] and exhaustive list of references therein. Fixed point theory has also been proven to be useful to describe the asymptotic behaviour, stability and equilibrium points of differential, functional, and difference equations and systems of equations, and continuous-time, discrete-time, and hybrid dynamic systems. See, for instance, [22-27] and references therein. Further links with technical results and some real-world examples are established through the paper related to the relevant problems of absolute stability and asymptotic hyperstability of continuous-time and discrete-time dynamic systems [1-8]. Such dynamic systems possess the significant physical property that their associate input-output energy is non-negative and finite for all time. Thus, they are purely dissipative systems, for a wide class of feedback nonlinear time-varying controllers satisfying an integral input-output inequality what leads to the global Lyapunov's stability for all controllers within such a class. Several operators are characterized but the most important one in the analysis is the one which maps the input space to the output space. Both such spaces are subspaces of a Hilbert space resulting to be, typically in real-world examples, either the space of square-integrable real or complex functions (or, in general, vector functions) or its corresponding squaresummable counterparts. The relevant property needed for a positive operator to be strictly positive is seen to be that its minimum modulus be nonzero so as to ensure that it is invertible if it is of a closed range. Note, on the other hand, 
that the crucial property for the boundedness and stability of the operator restricted to the Hilbert space of interest is that it will be stable on its whole definition domain.

\section{Problem Statement and Main Results}

Through this paper, one considers the complex Hilbert space $H$ on $\mathbf{C}$ and operators $G: H \rightarrow H$ and $K: H \rightarrow H$ which define the following associated relations:

$$
\begin{gathered}
y=G u+y_{h}, \\
u=K r-\varphi_{\Gamma}(y),
\end{gathered}
$$

where $y_{h}=y_{h}(p)$ and $p \in \mathbf{C}^{q}$ is some given complex parameterizing vector, and $r: \Gamma \rightarrow H \mid \operatorname{dom} K, \varphi_{\Gamma}: \Gamma \times$ $H \rightarrow \operatorname{ran} \varphi_{\Gamma}(\subset H), u: \Gamma \rightarrow(H \mid \operatorname{ran} K)+\operatorname{ran} \varphi_{\Gamma}(\subset H)$; and $y=y_{h}+y_{f}$, with $y_{h}: \Gamma \times \mathbf{C}^{q} \rightarrow H$ and $y_{f}: \Gamma \rightarrow H \mid$ ran $G$. The set $\Gamma$ is some appropriate domain to define the previous functions of interest. Examples which adjust to the previous structure are very common in the real world as, for instance, linear continuous-time dynamic systems (with $\Gamma=$ $\mathbf{R}_{0+}$ being the nonnegative real set for picking up values $t \epsilon$ $\Gamma$ of the continuous-time argument) and linear discrete-time dynamic systems (with $\Gamma=\mathbf{Z}_{0+}$ being the nonnegative integer set for values $t \in \Gamma$ of the discrete-time argument) where $r$ is an exogenous, or reference, signal, $u$ is a feedback control, $y_{h}$ is the parameterized response to initial conditions, versus $G u$ which is the forced response, $y$ is the measurable output to be controlled, and $\varphi_{\Gamma}(y)$ is a nonlinear (and, eventually, timevarying) controller device.

The inner products on the previous various Hilbert spaces are all denoted with the standard notation $\langle\cdot, \cdot\rangle$ and mutually distinguished easily depending on context without explicit notational subscripts referred to each concrete space. Assume that $\Gamma$ is an indicator set defining truncated elements of the Hilbert space as, for instance, a real interval or a subset of the nonnegative integers and $P_{t}$ is a projection operator being a truncation operator so that $x_{t}=P_{t} x$ and for each $t \in \Gamma$, and we define the seminorm on $H$ by $\|x\|_{t}=\left\|x_{t}\right\|=\left\|P_{t} x\right\|$; $\forall t \in \Gamma$ with $P_{t} \neq I$ and the family $\left\{\|\|_{t}: t \in \Gamma\right\}$ of seminorms defines the resolution topology on $H$ since $\left\{P_{t}: t \in H\right\}$ is a resolution of the identity [28]. Note that $\|x\|_{t_{1}} \geq\left\|x_{t_{2}}\right\|$ if $t_{1} \geq t_{2}$. For instance, if $\Gamma=\mathbf{R}_{0+}$ then $x(t)$ denotes a point value of $x: \mathbf{R}_{0_{+}} \rightarrow \mathbf{R}_{0+}$, for $t \in \Gamma$ while $x_{t}$ denotes the strip $x:[0, \mathrm{t}] \cap \mathbf{R}_{0+} \rightarrow \mathbf{R}_{0+}$. Through the paper the notation “*” stands for adjoint operators and also for complex conjugates of scalars or vectors depending on the context.

The problem to be discussed in the paper is the stability of (1) under an integral-type constraint for the controller specified later on, which characterizes a whole admissible class of controllers rather than an individual controller. Conditions are given for the positive feed-forward operator $G$ which is assumed to be bounded and of closed range is ensured to be also strictly positive, then boundedly invertible, with its existing inverse being also a strictly positive operator. If such an operator is bounded and strictly positive, then the inner products $\langle G u, u\rangle$ and $\left\langle G^{-1} u, u\right\rangle$ are both strictly positive and finitely upper-bounded for all nonzero input $u$.
The general formalism is given in Section 2 together with some links to contractive and pseudocontractive mappings while some real-world applications to asymptotic hyperstability of dynamic systems are then given in Section 3. The following preliminary result holds.

Proposition 1. Assume that $G: H \rightarrow H$ is a one-to-one linear operator with closed range. Then, the following properties hold

(i) $G: H \rightarrow H$ is invertible with nonzero minimum modulus,

(ii) if, in addition, $G: H \rightarrow H$ is positive (abbreviated notation being $G \geq 0)$, then $\langle G u, u\rangle>0$ for any nonzero $u: \Gamma \rightarrow H$,

(iii) there is $t \in \Gamma$ such that $\left\langle G P_{t} u, P_{t} u\right\rangle>0$ for any nonzero $u \in \operatorname{dom}(G)$.

Proof. Since $G$ on $H$ is one-to-one with closed range, it is also invertible from the open mapping theorem and then bounded below, so that there is $c \in \mathbf{R}_{+}$such that

$$
\|G u\|=\langle G u, G u\rangle^{1 / 2} \geq c\|u\|=c\langle u, u\rangle^{1 / 2} ; \quad \forall u \in \operatorname{dom} G .
$$

Then, the minimum modulus $\mu(G)$ of $G$ satisfies

$$
\mu(G)=\inf \left\{\frac{\|G u\|}{\|u\|}: u(\in \operatorname{dom} G) \neq 0\right\} \geq c>0
$$

and Property (i) has been proven. Now, if $G \geq 0$, then there is a self-adjoint operator $\widetilde{G}=\widetilde{G}^{*} \succeq 0$ on $X$ such that $G=$ $\widetilde{G}^{*} \widetilde{G}=\widetilde{G} \widetilde{G}^{*}=\widetilde{G}^{2}$ so that, since $\mu(\widetilde{G})>0$ from Property (i),

$$
\begin{aligned}
\langle G u, u\rangle^{1 / 2} & =\left\langle\widetilde{G}^{2} u, u\right\rangle^{1 / 2}=\langle\widetilde{G} u, \widetilde{G} u\rangle^{1 / 2} \\
& =\|\widetilde{G} u\|=\frac{\|\widetilde{G} u\|}{\|u\|}\|u\| \\
& \geq \inf \left\{\frac{\|\widetilde{G} u\|}{\|u\|}: u(\epsilon \operatorname{dom} G) \neq 0\right\}\|u\| \\
& \geq \mu(\widetilde{G})\|u\|>0 ; \quad \forall u(\neq 0): \Gamma \longrightarrow H
\end{aligned}
$$

and Property (ii) is proven.

(iii) Note that if, $u \neq 0$, then there is $t \in \Gamma$ such that $P_{t} u \neq 0$ and $\left\langle G P_{t} u, P_{t} u\right\rangle>0$ since one gets by Property (ii) that

$$
\begin{aligned}
\langle G u, u\rangle^{1 / 2} & =\sup _{t \in \Gamma}\left\langle G P_{t} u, P_{t} u\right\rangle^{1 / 2} \geq\left\langle G P_{t} u, P_{t} u\right\rangle^{1 / 2} \\
& =\left\langle\widetilde{G}^{2} P_{t} u, P_{t} u\right\rangle^{1 / 2}=\left\|\widetilde{G}\left(P_{t} u\right)\right\|=\frac{\left\|\widetilde{G}\left(P_{t} u\right)\right\|}{\left\|P_{t} u\right\|}\left\|P_{t} u\right\| \\
& \geq \inf \left\{\frac{\left\|\widetilde{G}\left(P_{t} u\right)\right\|}{\left\|P_{t} u\right\|}: u(\in \operatorname{dom} G) \neq 0\right\}\left\|P_{t} u\right\| \\
& \geq \mu(\widetilde{G})\left\|P_{t} u\right\|>0 ; \quad \forall u(\neq 0): \Gamma \longrightarrow H .
\end{aligned}
$$


Thus, $\left\langle G P_{t} u, P_{t} u\right\rangle>0$ for some $t \in \Gamma$ if $u \neq 0$. Hence, Property (iii) follows.

Definition 2. The operator $G: H \rightarrow H$ is said to be strictly positive (denoted as $G>0$ ) if it is positive (i.e., $G \geq 0$ ) and $\mu(G)>0$.

Note from Proposition 1 that if $G \geq 0$ is a one-to-one operator on $H$ with closed range, then it is invertible and $G>0$.

It is also direct to prove that Property (i) of Proposition 1 is equivalent to its given assumption so that one has [28].

Proposition 3. $G: H \rightarrow H$ is a one-to-one linear bounded operator with closed range if and only if it invertible with nonzero minimum modulus.

Proposition 4. If $G: H \rightarrow H$ is a one-to-one linear bounded strictly positive operator with closed range, then it is invertible and $G^{-1}: H \rightarrow H$ is also strictly positive with closed range and bounded and $\mu\left(G^{-1}\right)>0$ so that $\left\langle G^{-1} u, u\right\rangle>0$ for any nonzero $u: \Gamma \rightarrow H$.

Proof. Note that $1 /\left\|G^{-1}\right\|=\mu(G)>0$ from Proposition 1 . Thus, $\left\|G^{-1}\right\|<\infty$ so that $G^{-1}$ is bounded. Since $G$ is bounded, then $\|G\|<\infty$ and $1 /\|G\|=\mu\left(G^{-1}\right)>0$. Thus, $G^{-1}: H \rightarrow$ $H$ is also one-to-one with closed range from Proposition 3. Then, $G^{-1}=\widetilde{G}^{-1} \widetilde{G}^{*-1}=\widetilde{G}^{-2}$ is self-adjoint, and since $\mu\left(\widetilde{G}^{-1}\right)>0$, one has from Property (i) of Proposition 1 and Definition 2 that $G^{-1}>0$ since

$$
\begin{aligned}
&\left\langle G^{-1} u, u\right\rangle^{1 / 2}=\left\langle\widetilde{G}^{-2} u, u\right\rangle^{1 / 2}=\left\langle\widetilde{G}^{-1} u, \widetilde{G}^{-1} u\right\rangle^{1 / 2} \\
&=\left\|\widetilde{G}^{-1} u\right\|=\mu\left(G^{-1}\right)\|u\|>0, \\
& \forall u(\neq 0): \Gamma \longrightarrow H .
\end{aligned}
$$

Note that, if $G \succeq 0$, then $\langle G u, u\rangle$ can be zero for some nonzero $u: \Gamma \rightarrow H$. The following result refers to the fulfilment of relationships (1) for all $t \in \Gamma$, that is, on the space $H_{e}=\left\{f_{t}=P_{t} f:(f: \Gamma \rightarrow P C(\Gamma)) ; \forall t \in \Gamma\right\}$ provided that $G>0$ and bounded. Under some additional weak boundedness conditions, it is proven the stability of (1) with $u$ and $y$ belonging to $H$. Note that $H_{e}$ is not a Hilbert space (even though $H$ is a Hilbert space) since it is not ensured that, for any $f: \Gamma \rightarrow P C(\Gamma), f_{t}: \Gamma \rightarrow H$ as $(\Gamma \ni) t \rightarrow \infty$.

An important result follows.

Theorem 5. Assume that (1) holds for all $t \in \Gamma$, that is, $G$ : $H_{e} \rightarrow H_{e}$ and $K: H_{e} \rightarrow H_{e}$, where $H_{e}=\left\{f_{t}=P_{t} f:\right.$ $(f: \Gamma \rightarrow P C(\Gamma)) ; \forall t \in \Gamma\}, y_{h}=y_{h}(p)$ and $p \in \mathbf{C}^{q}$ is some given complex parameterizing vector, $r: \Gamma \rightarrow H_{e} \mid \operatorname{dom} K$, $\varphi_{\Gamma}: \Gamma \times H_{e} \rightarrow \operatorname{ran} \varphi_{\Gamma}, u: \Gamma \rightarrow\left(H_{e} \mid \operatorname{ran} K\right)+\operatorname{ran} \varphi_{\Gamma} ;$ and $y=y_{h}+y_{f}$, with $y_{h}: \Gamma \times \mathbf{C}^{q} \rightarrow H$ and $y_{f}: \Gamma \rightarrow H_{e} \mid$ ran $G$. Assume also that

(1) $y_{h}=y_{h}(p)$ is bounded and $P_{t} y_{h} \rightarrow 0$ as $(\Gamma \ni) t \rightarrow$ $\infty$,
(2) $G: H_{e} \rightarrow H_{e}$ is stable (or, equivalently, $G: H_{e} \mid H \rightarrow$ $H$ is bounded and causal), one-to-one, and with closed range,

(3) $G>0$,

(4) $\mathrm{K}: \mathrm{H} \rightarrow \mathrm{H}$ is bounded,

(5) $r: \Gamma \rightarrow H$ is bounded,

(6) $\left\langle P_{t} y, P_{t}\left(\varphi_{\Gamma}(y)\right)\right\rangle \geq-\gamma_{t} \geq-\gamma>-\infty ; \forall t \in \Gamma$.

Then, $u, y_{f}, y: \Gamma \rightarrow H$, and they are bounded. Also, if $r \equiv 0$, then $u(t) \rightarrow 0, y_{f}(t) \rightarrow 0, y(t) \rightarrow 0$ as $(\Gamma \ni) t \rightarrow \infty$.

Proof. Direct calculations yield

$$
\begin{aligned}
\langle y, u\rangle= & \left\langle G u+y_{h}, K r-\varphi_{\Gamma}(y)\right\rangle \\
= & \langle G u, K r\rangle+\left\langle y_{h}, K r\right\rangle \\
& -\left(\langle G u, \varphi(y)\rangle+\left\langle y_{h}, \varphi_{\Gamma}(y)\right\rangle\right) \\
= & \langle G u, K r\rangle+\left\langle y_{h}, K r\right\rangle+\langle G u, u\rangle \\
& -\left\langle y_{h}, \varphi_{\Gamma}(y)\right\rangle-\langle G u, K r\rangle \\
= & \langle G u, u\rangle+\left\langle y_{h}, K r-\varphi_{\Gamma}(y)\right\rangle \\
\geq & \mu^{2}(\widetilde{G})\|u\|^{2}+\left\langle y_{h}, K r-\varphi_{\Gamma}(y)\right\rangle \\
= & \mu^{2}(\widetilde{G}) \sup _{t \in \Gamma}\left\|P_{t} u\right\|^{2}+\left\langle y_{h}, K r-\varphi_{\Gamma}(y)\right\rangle \\
\geq & \mu^{2}(\widetilde{G})\left\|P_{t} u\right\|^{2}+\left\langle y_{h}, K r-\varphi_{\Gamma}(y)\right\rangle ; \quad \forall t \in \Gamma
\end{aligned}
$$

since $u=K r-\varphi_{\Gamma}(t, y)$ and $\langle G u, u\rangle \geq \mu^{2}(\widetilde{G})\|u\|^{2} \geq$ $\mu^{2}(\widetilde{G})\left\|P_{t} u\right\|^{2}>0$ for any nonzero control from Proposition 1 . One gets in the same way that

$$
\left\langle P_{t} y, P_{t} u\right\rangle \geq \mu^{2}(\widetilde{G})\left\|P_{t} u\right\|^{2}+\left\langle P_{t}\left(y_{h}\right), P_{t}\left(K r-\varphi_{\Gamma}(y)\right)\right\rangle,
$$

Since $\left\langle P_{t} y, P_{t}\left(\varphi_{\Gamma}(y)\right)\right\rangle \geq-\gamma_{t}>-\infty ; \forall t \in \Gamma$, one gets also that

$$
\begin{aligned}
&\langle y, u\rangle=\left\langle y, K r-\varphi_{\Gamma}(y)\right\rangle=\langle y, K r\rangle-\left\langle y, \varphi_{\Gamma}(y)\right\rangle \\
& \leq\langle y, K r\rangle+\sup _{t \in \Gamma} \gamma_{t} ; \quad \forall t \in \Gamma, \\
&\left\langle P_{t} y, P_{t} u\right\rangle \leq\left\langle P_{t} y, P_{t}(K r)\right\rangle+\gamma_{t} ; \quad \forall t \in \Gamma .
\end{aligned}
$$

One gets from (7), (9), (8), and (10) that

$$
\begin{aligned}
&\langle y, K r\rangle+\gamma \geq \mu^{2}(\widetilde{G})\|u\|^{2} \\
&+\left\langle y_{h}, K r-\varphi_{\Gamma}(y)\right\rangle \\
& \geq \mu^{2}(\widetilde{G})\left\|P_{t} u\right\|^{2}+\left\langle y_{h}, K r-\varphi_{\Gamma}(y)\right\rangle, \\
& \forall t \in \Gamma, \quad(11) \\
&\left\langle P_{t} y, P_{t}(K r)\right\rangle \gamma_{t} \geq \mu^{2}(\widetilde{G})\left\|P_{t} u\right\|^{2}\left\langle P_{t}\left(y_{h}\right), P_{t}\left(K r-\varphi_{\Gamma}(y)\right)\right\rangle ; \\
& \forall t \in \Gamma,
\end{aligned}
$$


where $0<\gamma=\sup _{t \in \Gamma} \gamma_{t}<\infty$. Now, since $K$ is a bounded operator, $r$ is a bounded function, $P_{t} y_{h} \rightarrow 0$ as $t \rightarrow \infty$, and $G>0$ is bounded and one-to-one with closed range so that $\widetilde{G}>0$ is also bounded and one-to-one with closed range implying from Proposition 1 that $\mu(G)=\mu^{2}(\widetilde{G})>0$, and one gets from (12) that

$$
\begin{array}{r}
\liminf _{\Gamma \ni t \rightarrow \infty}\left[\gamma_{t}+\left|\left\langle P_{t}\left(G P_{t} u\right)\right\rangle, P_{t}(K r)\right\rangle \mid\right. \\
\left.-\mu^{2}(\widetilde{G})\left\|P_{t} u\right\|^{2}\right] \\
\geq \liminf _{\Gamma \ni t \rightarrow \infty}\left[\gamma_{t}+\left\langle P_{t}\left(G P_{t} u\right)\right\rangle, P_{t}(K r)\right. \\
\left.>-\mu^{2}(\widetilde{G})\left\|P_{t} u\right\|^{2}\right] \geq 0 .
\end{array}
$$

Assume that there is some unbounded $u: \Gamma \rightarrow H$. Then, the subsequent contradiction

$$
0 \leq \liminf _{\Gamma \ni t \rightarrow \infty}\left[\gamma_{t}+\mu^{2}(\widetilde{G})\left(\lambda-\left\|P_{t} u\right\|\right)\left\|P_{t} u\right\|\right]=-\infty
$$

follows from (13) for some $\lambda \in \mathbf{R}_{0+}$ since $\mu(\widetilde{G})>0$. Then any $u: \Gamma \rightarrow H$ is bounded. Since the operator $G$ on $H_{e}$ is bounded, it is stable, and then $G: H_{e} \mid H \rightarrow H$ is also bounded and causal, and, since the function $u: \Gamma \rightarrow H$ is bounded, then $y_{f}: \Gamma \rightarrow H$ is also bounded with $\left\|y_{f}\right\| \leq$ $\|G\|\|u\|$ and $\left\|P_{t} y_{f}\right\| \leq\left\|P_{t} G P_{t}\right\|\|u\| \leq\|G\|\|u\| ; \forall t \in \Gamma$, and $y: \Gamma \rightarrow H$ is also bounded since $y=y_{h}+y_{f}$. On the other hand, if $r: \Gamma \rightarrow H$ is identically zero, then one gets from (13) $0 \leq \mu(\widetilde{G}) \lim \sup _{\Gamma \ni t \rightarrow \infty}\left\|P_{t} u\right\| \leq \gamma<\infty$, and, since $\mu(\widetilde{G})>0$, then $\exists \lim _{\Gamma \ni t \rightarrow \infty} u(t)=0$.

Also, it is clear that, since $u: \Gamma \rightarrow H_{e}$, and since $u$ : $\Gamma \rightarrow H$ is bounded and converges asymptotically to zero and $\left\|y_{f}\right\| \leq\|G\|\|u\|$, then $u: \Gamma \rightarrow H, y_{f}$ is bounded, $y_{f}: \Gamma \rightarrow$ $H$, and then $\exists \lim _{\Gamma \ni t \rightarrow \infty} y(t)=\lim _{\Gamma \ni t \rightarrow \infty} y_{f}(t)=0$ since $y_{h}: \Gamma \rightarrow H$ is bounded and asymptotically vanishing.

The assumption 6 of Theorem 5 can be relaxed leading to the following stronger result.

Corollary 6. Theorem 5 holds if its assumption 6 is relaxed to $\liminf _{t \rightarrow \infty}\left\langle P_{t} y, P_{t}\left(\varphi_{\Gamma}(y)\right)\right\rangle \geq-\gamma>-\infty$.

Proof. Note that (7) still holds since it is independent of assumption 6. The constraint (12) is modified as follows:

$$
\begin{gathered}
\liminf _{\Gamma \ni t \rightarrow \infty}\left\langle P_{t} y, P_{t}(K r)\right\rangle+\gamma_{t}-\mu^{2}(\widetilde{G})\left\|P_{t} u\right\|^{2} \\
-\left\langle P_{t}\left(y_{h}\right), P_{t}\left(K r-\varphi_{\Gamma}(y)\right)\right\rangle \geq 0
\end{gathered}
$$

which makes (13) to remain valid, and Theorem 5 still holds.

In a physical context, $E=\langle y, u\rangle$ is the whole input-output energy of (1), $E(t)=\left\langle P_{t} y, P_{t} u\right\rangle$ is the input-output energy dissipated on $[0, t] \cap \Gamma$, and $(u * y)(t)$ is the instantaneous input-output power at $t \in \Gamma$ while $\left\langle G u+y_{h}, K r\right\rangle$ is the energy supplied by the external source. Particular cases of interest in control engineering are (a) if the reference input $r \equiv 0$, then the feedback control system is a regulator evolving only from its initial conditions, (b) if such reference is a constant real level, then the control system is a position servomechanism, (c) if the reference $r(t)=K t$ for $t \in \Gamma$, then the control system is a velocity servomechanism and so forth.

On the other hand, the extended Popov-type control inequality of the controller $\left\langle P_{t} y, P_{t}\left(\varphi_{\Gamma}(y)\right)\right\rangle \geq-\gamma>-\infty$ and $G>0$ implies that $0 \leq E(t) \leq \gamma<\infty,(0<E \leq \gamma<\infty$ for any nonzero control input with compact support); $\forall t \in \Gamma$ and all $\varphi_{\Gamma}(y)$ satisfying the assumption 6 of Theorem 5 ; that is the input-output energy is nonnegative and bounded; $\forall t \in \Gamma$. The use of such a constraint allows the simultaneous investigation of the maintenance of the positivity and stability properties of (1) under a class of nonlinear time-varying controllers (defined by such a Popov constraint itself) rather than for a particular controller device belonging to such a class.

Note that $G$ on $H_{e}$ is stable since $\|G u\|_{t} \leq M\|u\|_{t}$ for some finite $\in \mathbf{R}_{+} ; \forall t \in \Gamma$ and, equivalently, $G: H_{e} \mid H \rightarrow$ $H$ is bounded. Now, one concludes from Proposition 4 for the system defined by the inverse operator $G^{-1}$ that $0 \leq$ $\left\langle P_{t}\left(G^{-1} u\right), P_{t} u\right\rangle<\infty$ for any admissible control input $u$ since $G^{-1}>0$, bounded and causal.

The following result basically reformulates Theorem 5 if $G \quad H$ is a strictly positive pseudocontraction. Since the contribution of initial conditions and a bounded exogenous reference do not modify the stability properties, as seen from Theorem 5, they are assumed to be null in the sequel.

Theorem 7. Assume that the relationships of (1) hold for all $t \in \Gamma$ with $r \equiv 0, y_{h} \equiv 0, G: H_{e} \rightarrow H_{e}, \varphi_{\Gamma}: \Gamma \times H_{e} \rightarrow \operatorname{ran} \varphi_{\Gamma}$ and, furthermore,

(1) $G: H_{e} \mid H \rightarrow H$ is bounded and causal, one-to-one, and with closed range.

(2) $G>0$.

(3) $\left\langle P_{t} y, P_{t}\left(\varphi_{\Gamma}(y)\right)\right\rangle \geq-\gamma_{t} \geq-\gamma>-\infty ; \forall t \in \Gamma$.

Then, $u, y_{f}, y: \Gamma \rightarrow H$ and are bounded, and $u(t) \rightarrow 0$, $y(t) \rightarrow 0$ as $(\Gamma \ni) t \rightarrow \infty$. Furthermore, one gets for any, $u_{1}, u_{2}: \Gamma \rightarrow \operatorname{ran} \varphi_{\Gamma}$ that

$$
\left\langle u_{1}, u_{2}\right\rangle+\left\langle u_{1}, u_{2}\right\rangle^{*} \leq \frac{2 \gamma}{\mu^{2}(\widetilde{G})}
$$

If, in addition, $G \mid H$ is a pseudocontraction, then

$$
0 \leq\left\langle G u_{1}-G u_{2}, u_{1}-u_{2}\right\rangle \leq \gamma \min \left(1, \frac{4}{\mu^{2}(\widetilde{G})}\right)
$$

with the lower-bound equating zero if and only if $u_{1}=u_{2}$.

Proof. Take the relation proved in Theorem $5\langle y, u\rangle=\langle G u+$ $\left.y_{h}, K r-\varphi_{\Gamma}(y)\right\rangle$ under zero exogenous reference and initial conditions in (1) to yield

$$
0<\mu^{2}(\widetilde{G})\langle u, u\rangle \leq\langle G u, u\rangle=\langle\widetilde{G} u, \widetilde{G} u\rangle \leq \gamma<\infty .
$$


Since $G>0$ then $G=G^{*}$, and one gets for $u=u_{1}-u_{2}$

$$
\begin{aligned}
0 & \leq\left\langle G u_{1}-G u_{2}, u_{1}-u_{2}\right\rangle=\left\langle\widetilde{G} u_{1}-\widetilde{G} u_{2}, \widetilde{G} u_{1}-\widetilde{G} u_{2}\right\rangle \\
& =\left\langle\widetilde{G} u_{1}, \widetilde{G} u_{1}\right\rangle+\left\langle\widetilde{G} u_{2}, \widetilde{G} u_{2}\right\rangle-\left\langle\widetilde{G} u_{1}, \widetilde{G} u_{2}\right\rangle-\left\langle\widetilde{G} u_{2}, \widetilde{G} u_{1}\right\rangle \\
& =\left\langle G u_{1}, u_{1}\right\rangle+\left\langle G u_{2}, u_{2}\right\rangle-\left\langle G u_{1}, u_{2}\right\rangle-\left\langle G u_{2}, u_{1}\right\rangle \\
& \leq 2 \gamma-\left\langle G u_{1}, u_{2}\right\rangle-\left\langle u_{2}, G u_{1}\right\rangle \\
& =2 \gamma-\left\langle G u_{1}, u_{2}\right\rangle-\left\langle G u_{1}, u_{2}\right\rangle^{*} \\
& \Rightarrow \\
0 & \leq \max \left(0,\left\langle G u_{1}, u_{2}\right\rangle+\left\langle G u_{1}, u_{2}\right\rangle^{*}\right) \\
& \leq\left\langle G u_{1}-G u_{2}, u_{1}-u_{2}\right\rangle-\left\langle G u_{1}, u_{2}\right\rangle-\left\langle G u_{1}, u_{2}\right\rangle^{*} \\
& \leq 2 \gamma<\infty \\
0 & \leq\left\|u_{1}-u_{2}\right\|^{2}=\left\langle u_{1}-u_{2}, u_{1}-u_{2}\right\rangle \\
& =\left\|u_{1}\right\|^{2}+\left\|u_{2}\right\|^{2}-\left\langle u_{1}, u_{2}\right\rangle-\left\langle u_{1}, u_{2}\right\rangle^{*} \\
& \leq \frac{2 \gamma}{\mu^{2}(\widetilde{G})}-\left\langle u_{1}, u_{2}\right\rangle-\left\langle u_{1}, u_{2}\right\rangle^{*}
\end{aligned}
$$

and $\left\langle u_{1}, u_{2}\right\rangle+\left\langle u_{1}, u_{2}\right\rangle^{*} \leq 2 \gamma / \mu^{2}(\widetilde{G})$. Assume that $G>0$ is, furthermore, a pseucontraction on $H$. Then,

$$
\begin{aligned}
0 & \leq\left\langle G u_{1}-G u_{2}, u_{1}-u_{2}\right\rangle \\
& =\left\langle\widetilde{G} u_{1}-\widetilde{G} u_{2}, \widetilde{G} u_{1}-\widetilde{G} u_{2}\right\rangle \leq\left\|u_{1}-u_{2}\right\|^{2}
\end{aligned}
$$

and, equivalently,

$$
\begin{gathered}
\left\|G u_{1}-G u_{2}\right\|^{2} \leq\left\|u_{1}-u_{2}\right\|^{2}+\left\|(I-G) u_{1}-(I-G) u_{2}\right\|^{2} \\
0 \leq\left\langle G u_{1}-G u_{2}, u_{1}-u_{2}\right\rangle \leq\left\langle\widetilde{G} u_{1}-\widetilde{G} u_{2}, \widetilde{G} u_{1}-\widetilde{G} u_{2}\right\rangle \\
\quad \leq\left\|u_{1}-u_{2}\right\|^{2} \leq \frac{2 \gamma}{\mu^{2}(\widetilde{G})}-\left\langle u_{1}, u_{2}\right\rangle-\left\langle u_{1}, u_{2}\right\rangle^{*}
\end{gathered}
$$

implies that

$$
\begin{aligned}
\left\langle u_{1}, u_{2}\right\rangle & +\left\langle u_{1}, u_{2}\right\rangle^{*} \\
< & \left\langle G u_{1}-G u_{2}, u_{1}-u_{2}\right\rangle \\
& +\left\langle u_{1}, u_{2}\right\rangle+\left\langle u_{1}, u_{2}\right\rangle^{*} \leq \frac{2 \gamma}{\mu^{2}(\widetilde{G})}
\end{aligned}
$$

and the following cases can occur.

(a) $0 \leq\left\langle G u_{1}-G u_{2}, u_{1}-u_{2}\right\rangle \leq \min \left(\gamma, 2 \gamma / \mu^{2}(\widetilde{G})-\left\langle u_{1}, u_{2}\right\rangle-\right.$ $\left.\left\langle u_{1}, u_{2}\right\rangle^{*}\right)$ if the controls $u_{1}$ and $u_{2}$ fulfil $0<\left\langle u_{1}, u_{2}\right\rangle+$ $\left\langle u_{1}, u_{2}\right\rangle^{*}<2 \gamma / \mu^{2}(\widetilde{G})$.

(b) $0 \leq\left\langle G u_{1}-G u_{2}, u_{1}-u_{2}\right\rangle \leq \min \left(\gamma, 2 \gamma / \mu^{2}(\widetilde{G})-\right.$ $\left.\left|\left\langle u_{1}, u_{2}\right\rangle+\left\langle u_{1}, u_{2}\right\rangle^{*}\right|\right)$ if the controls $u_{1}$ and $u_{2}$ fulfil $\left\langle u_{1}, u_{2}\right\rangle+\left\langle u_{1}, u_{2}\right\rangle^{*}<0$. (c) $0<2 \gamma / \mu^{2}(\widetilde{G}) \leq\left\langle G u_{1}-G u_{2}, u_{1}-u_{2}\right\rangle \leq \gamma$ if the controls $u_{1}$ and $u_{2}$ fulfil $\left\langle u_{1}, u_{2}\right\rangle+\left\langle u_{1}, u_{2}\right\rangle^{*} \geq$ $2 \gamma / \mu^{2}(\widetilde{G})$. This case is only feasible with equality.

Combining the three cases one gets that

$$
0 \leq\left\langle G u_{1}-G u_{2}, u_{1}-u_{2}\right\rangle \leq \gamma \min \left(1, \frac{4}{\mu^{2}(\widetilde{G})}\right)
$$

with the lower-bound equating zero if and only if $u_{1}=u_{2}$; that is $u=u_{1}-u_{2}=0$.

Basically, Theorem 7 states that a strictly positive operator, which is also a pseudocontraction, subject to a feedback control law satisfying a Popov-type inequality keeps the boundedness of the input-output energy with a modified upper-bound which improves that associated to the Popov inequality if the minimum modulus of $G$ satisfies $\mu(G)>4$. The following result guarantees the fulfilment of Theorem 5 if $G: H \rightarrow H$ is strictly positive and asymptotically pseudocontractive in the intermediate sense under a modified Popov-type inequality.

Theorem 8. Assume that

(1) $G>0$ is one-to-one, bounded, causal, and of closed range with minimum modulus $\mu(G)>\alpha$,

(2) $G: H \rightarrow H$ is asymptotically pseudocontractive in the intermediate sense satisfying the constraint,

$$
\begin{array}{r}
0 \leq\left\langle P_{t} \bar{y}_{1}-P_{t} \bar{y}_{2}, P_{t} \bar{u}_{1}-P_{t} \bar{u}_{2}\right\rangle \leq \alpha_{t}\left\|P_{t} \bar{u}_{1}-P_{t} \bar{u}_{2}\right\|^{2} ; \\
\forall t \in \Gamma
\end{array}
$$

for some real convergent sequence $\left\{\alpha_{t}\right\}_{t \in \Gamma}$ in $[\alpha, \infty)$ such that $\alpha_{t} \rightarrow \alpha \in(0,1]$ as $t(\in \Gamma) \rightarrow \infty$ and zero initial conditions and exogenous reference in (1), where

$$
\begin{aligned}
& \bar{y}_{t}=y_{t+T}-y_{t}=P_{t+T} y-P_{t} y, \\
& \bar{u}_{t}=u_{t+T}-u_{t}=P_{t+T} u-P_{t} u
\end{aligned}
$$

are incremental values of $y$ and $u$ with $t, t+T(>t) \in \Gamma$ being adjacent elements in the strict ordering on $\Gamma$ if such an indexing set is discrete and $[t, t+T]$ being $a$ closed interval of nonzero constant Lebesgue measure $T$ in $\Gamma$ if such an indexing set is real,

(3) the following inequality holds:

$$
\liminf _{\Gamma \ni t \rightarrow \infty}\left(\left\langle P_{t} y, P_{t}\left(\varphi_{\Gamma}(\bar{y})\right)\right\rangle+\alpha_{t}\|\bar{u}\|_{t}^{2}\right) \geq 0 .
$$

Then, $u, y: \Gamma \rightarrow H$, and they are bounded, and, furthermore, $u(t) \rightarrow 0, y(t) \rightarrow 0$ as $(\Gamma \ni) t \rightarrow \infty$ under a zero exogenous input and initial conditions.

Proof. Since $G: H \rightarrow H$ is asymptotically pseudocontractive in the intermediate sense

$$
\begin{aligned}
0 & \leq\left\langle P_{t} \bar{y}_{1}-P_{t} \bar{y}_{2}, P_{t} \bar{u}_{1}-P_{t} \bar{u}_{2}\right\rangle \\
& =\left\langle P_{t} G P_{t} \bar{u}_{1}-P_{t} G P_{t} \bar{u}_{2}, P_{t} \bar{u}_{1}-P_{t} \bar{u}_{2}\right\rangle \\
& \leq \alpha_{t}\left\|P_{t} \bar{u}_{1}-P_{t} \bar{u}_{2}\right\|^{2} ; \quad \forall t \in \Gamma
\end{aligned}
$$


for zero initial conditions and exogenous reference in (1). Note that these particular conditions do not modify the boundedness-type stability properties related to the injection of any bounded exogenous reference under bounded initial conditions and some real convergent sequence $\left\{\alpha_{t}\right\}_{t \in \Gamma}$ in $[\alpha, \infty)$ such that $\alpha_{t} \rightarrow \alpha \in(0,1)$ as $t(\in \Gamma) \rightarrow \infty$. Since

$$
\begin{aligned}
& \liminf _{\Gamma \ni t \rightarrow \infty}\left(\left\langle P_{t} \bar{y}, P_{t}\left(\varphi_{\Gamma}(\bar{y})\right)\right\rangle+\alpha_{t}\|\bar{u}\|_{t}^{2}\right) \\
& \quad=\liminf _{\Gamma \ni t \rightarrow \infty}\left(\alpha_{t}\left\|P_{t} \bar{u}\right\|^{2}-\left\langle P_{t} \bar{y}, P_{t} \bar{u}\right\rangle\right) \geq 0,
\end{aligned}
$$

then one has

$$
\begin{aligned}
0 & \leq \mu^{2}(\widetilde{G})\left\|\bar{u}_{t}\right\|^{2}=\mu^{2}(\widetilde{G})\|\bar{u}\|_{t}^{2} \leq \alpha_{t}\|\bar{u}\|_{t}^{2} \\
& \Longrightarrow\left(-\alpha_{t}\|\bar{u}\|_{t}^{2} \leq\left(\mu^{2}(\widetilde{G})-\alpha_{t}\right)\|\bar{u}\|_{t}^{2} \leq 0\right),
\end{aligned}
$$

$$
\forall t \in \Gamma
$$

so that, if $\alpha=\lim _{\Gamma \ni t \rightarrow \infty} \alpha_{t}<\mu^{2}(\widetilde{G})$, then $\lim \sup _{\Gamma \ni t \rightarrow \infty}\left(\mu^{2}(\widetilde{G})-\alpha_{t}\right)\|\bar{u}\|_{t}^{2} \leq 0$ and $u(t) \rightarrow 0$ as $\Gamma \in t \rightarrow$ $\infty . u: \Gamma \rightarrow H$ is bounded since it is piecewise continuous with eventual bounded discontinuities, and $T>0$ and finite. Since $G$ on $H_{e}$ and $G$ restricted to $H$ are stable, $y: \Gamma \rightarrow H$ is also bounded and converges to zero.

A particular case of Theorem 8 of interest is as follows.

Corollary 9. Theorem 8 holds if the assumption 2 is replaced by $G: H \rightarrow H$ being a pseudocontraction.

Proof. It follows since Theorem 8 holds, in particular, under the condition $\alpha_{t}=\alpha=1 ; \forall t \in \Gamma$.

If $G: H \rightarrow H$ is strictly positive and contractive, we obtain the subsequent result.

Theorem 10. Assume that

(1) $G>0$ is one-to-one, bounded, causal, and of closed range.

(2) $G: H \rightarrow H$ satisfies the following positive-bounded and contractive constraints for some given $\beta \in \Gamma$ and $u=u_{1}-u_{2}$ :

$$
\begin{aligned}
0 \leq & \left\langle P_{\beta} G P_{\beta} u_{1}-P_{\beta} G P_{\beta} u_{2}, P_{\beta} u_{1}-P_{\beta} u_{2}\right\rangle \\
& -\left\langle P_{0} G P_{0} u_{1}-P_{0} G P_{0} u_{2}, P_{0} u_{1}-P_{0} u_{2}\right\rangle \leq M<\infty \\
0 \leq & \left\langle P_{t+2 \beta} G P_{t+2 \beta} u_{1}-P_{t+2 \beta} G P_{t+2 \beta} u_{2}, P_{t+2 \beta} u_{1}-P_{t+2 \beta} u_{2}\right\rangle \\
& -\left\langle P_{t+\beta} G P_{t+T} u_{1}-P_{t+\beta} G P_{t+\beta} u_{2}, P_{t+\alpha} u_{1}-P_{t+\beta} u_{2}\right\rangle \\
\leq & \chi_{t}\left\langle P_{t+\beta} G P_{t+\phi} u_{1}-P_{t+\beta} G P_{t+\beta} u_{2}, P_{t+\beta} u_{1}-P_{t+\beta} u_{2}\right\rangle \\
& -\chi_{t}\left\langle P_{t} G P_{t} u_{1}-P_{t} G P_{t} u_{2}, P_{t} u_{1}-P_{t} u_{2}\right\rangle ; \quad \forall t \in \Gamma
\end{aligned}
$$

with $\left\{\chi_{t}\right\}_{t \in \Gamma}$ being a real sequence subject to $0 \leq \chi_{t} \leq$ $\sigma<\infty$ and $\prod_{\Gamma \ni t}^{t+\beta}\left[\chi_{t}\right]=\chi<1$ with $\beta,(t+n \beta) \epsilon$ $\Gamma ; \forall n \in \mathbf{Z}_{+}, \forall t \in \Gamma$ provided that $0 \in \Gamma$ is the first element of $\Gamma$, and (3) $\left\langle P_{t} y, P_{t}\left(\varphi_{\Gamma}(y)\right)\right\rangle \geq-\gamma_{t} \geq-\gamma=M /(\chi-1)>-\infty$;
$\forall t \in \Gamma$.

Then, $u, y: \Gamma \rightarrow H$, and they are bounded, and, furthermore, $u(t) \rightarrow 0, y(t) \rightarrow 0$ as $(\Gamma \ni) t \rightarrow \infty$ under a zero exogenous input and initial conditions.

Proof. Note from (30) that

$$
\begin{gathered}
0 \leq\left\langle P_{n \beta} G P_{n \beta} u_{1}-P_{n \beta} G P_{n \beta} u_{2}, P_{(n-1) \beta} u_{1}-P_{(n-1) \beta} u_{2}\right\rangle \\
\leq \chi^{n-1}\left(\left\langle P_{\beta} G P_{\beta} u_{1}-P_{\beta} G P_{\beta} u_{2}, P_{\beta} u_{1}-P_{\beta} u_{2}\right\rangle\right. \\
\left.\quad-\left\langle P_{0} G P_{0} u_{1}-P_{0} G P_{0} u_{2}, P_{0} u_{1}-P_{0} u_{2}\right\rangle\right) \\
\quad \leq M<\infty
\end{gathered}
$$

since $n \beta \in \Gamma ; \forall n \in \mathbf{Z}_{+}$, and $0 \in \Gamma$ is the first element of $\Gamma$. Thus, since $0 \leq \chi<1$, one gets

$$
\begin{aligned}
0 & \leq \sum_{n \in \mathbf{Z}_{+}}\left\langle P_{n \beta} G P_{n \beta} u_{1}-P_{n \beta} G P_{n \beta} u_{2}, P_{(n-1) \beta} u_{1}-P_{(n-1) \beta} u_{2}\right\rangle \\
& \leq\left\langle G u_{1}-G u_{2}, u_{1}-u_{2}\right\rangle \leq \gamma=\frac{M}{1-\chi}<\infty
\end{aligned}
$$

and also

$$
\begin{aligned}
0 & \leq \sum_{n=1}^{N}\left\langle P_{n \beta} G P_{n \beta} u_{1}-P_{n \beta} G P_{n \beta} u_{2}, P_{(n-1) \beta} u_{1}-P_{(n-1) \beta} u_{2}\right\rangle \\
& \leq\left\langle G u_{1}-G u_{2}, u_{1}-u_{2}\right\rangle \leq \gamma=\frac{M}{1-\chi}<\infty, \quad \forall N \in \mathbf{Z}_{+} .
\end{aligned}
$$

Thus, $u(t)=u_{1}(t)-u_{2}(t) \rightarrow 0$ as $\Gamma \in t \rightarrow \infty$. Since $u \equiv 0$ is an admissible control, one concludes that any admissible control is bounded and it converges asymptotically to zero. The output $y(t)$ has a similar property.

\section{Application Examples}

Example 1. Define the truncated function within the time interval $[0, t] ; \forall t \in \mathbf{R}_{0+}$ of $f: \mathbf{R} \rightarrow \mathbf{R}$ as follows:

$$
f_{t}(\tau)=P_{t} f= \begin{cases}f(\tau) & \text { for } \tau \in[0, t] \\ 0 & \text { otherwise. }\end{cases}
$$

Thus, the output of a single-input single-output linear timeinvariant continuous-time dynamic system of $n$th order and initial state $x(0)=x_{0} \in \mathbf{R}^{n}$ under a piecewise continuous control with eventual isolated bounded discontinuities $u$ : $\mathbf{R} \rightarrow \mathbf{R} \cap H$, where $H=L^{2}\left(\mathbf{R}_{0+}\right) \equiv L^{2}[0, \infty)$ the Hilbert space of the square-integrable functions on $\mathbf{R}_{0+}$ is

$$
\begin{aligned}
y(t) & =\int_{0}^{t} g(t, \tau) u(\tau) d \tau+c\left(t, x_{0}\right) \\
& =\int_{-\infty}^{\infty} g(t, \tau) u_{t}(\tau) d \tau+c\left(t, x_{0}\right) \\
& =(g * u)(t)+c\left(t, x_{0}\right), \quad \forall t \in \mathbf{R}_{0+},
\end{aligned}
$$


where $\Gamma=\mathbf{R}_{0+}=\{z \in \mathbf{R}: z \geq 0\}, g: \mathbf{R} \times \mathbf{R} \rightarrow \mathbf{R}$ is the impulse response, $c\left(t, x_{0}\right)$ is the zero-input response (i.e., the response contribution due to initial conditions) for initial sate $x_{0}$, and “*” stands for the convolution integral operator. Since the dynamic system is realizable, $g(t, \tau)=0$ for $\tau>t$. The complex function $G: \mathbf{C} \rightarrow \mathbf{C}$ defined as $G(s)=\mathbf{L}(g(t))$ is the transfer function, where $\mathbf{L}$ stands for the Laplace transform of the impulse response where it exists. After defining $y(t)=0$ for $t<0$, the input-output energy obeys the following relations by using twice Parseval theorem:

$$
\begin{aligned}
& E(t)=\int_{0}^{t} y(\tau) u(\tau) d \tau=\int_{-\infty}^{\infty} y(\tau) u_{t}(\tau) d \tau \\
& =\frac{1}{2 \pi} \int_{-\infty}^{\infty} Y(\mathbf{i} \omega) U_{t}(-\mathbf{i} \omega) d \omega=\langle y, u\rangle_{t}=\left\langle y, u_{t}\right\rangle \\
& =\left\langle y_{t}, u_{t}\right\rangle=\left\langle y_{t}, u\right\rangle=\frac{1}{2 \pi}\left\langle Y, U_{t}\right\rangle \\
& =\frac{1}{2 \pi} \int_{-\infty}^{\infty} G(\mathbf{i} \omega) U_{t}(\mathbf{i} \omega) U_{t}(-\mathbf{i} \omega) d \omega \\
& +\int_{0}^{t} c\left(\tau, x_{0}\right) u(\tau) d \tau \\
& =\frac{1}{2 \pi} \int_{-\infty}^{\infty} G(\mathbf{i} \omega)\left|U_{t}(\mathbf{i} \omega)\right|^{2} d \omega \\
& +\int_{0}^{t} c\left(\tau, x_{0}\right) u(\tau) d \tau \\
& =\frac{1}{2 \pi}\left\langle G U_{t}, U_{t}\right\rangle+\int_{0}^{t} c\left(\tau, x_{0}\right) u(\tau) d \tau \\
& =\frac{1}{2 \pi}\left\langle G,\left|U_{t}\right|^{2}\right\rangle+\int_{0}^{t} c\left(\tau, x_{0}\right) u(\tau) d \tau \\
& \geq \frac{1}{2 \pi}\left(\min _{\omega \in \mathbf{R}_{0+}} \operatorname{Re} G(\mathbf{i} \omega)\right) \int_{-\infty}^{\infty}\left|U_{t}(\mathbf{i} \omega)\right|^{2} d \omega \\
& =\left(\min _{\omega \in \mathbf{R}_{0+}} \operatorname{Re} G(\mathbf{i} \omega)\right) \int_{-\infty}^{\infty}\left|u_{t}(\tau)\right|^{2} d \tau \\
& +\int_{0}^{t} c\left(\tau, x_{0}\right) u(\tau) d \tau \\
& =\left(\min _{\omega \in \mathbf{R}_{0+}} \operatorname{Re} G(\mathbf{i} \omega)\right) \int_{0}^{t}|u(\tau)|^{2} d \tau \\
& +\int_{0}^{t} c\left(\tau, x_{0}\right) u(\tau) d \tau \\
& =\left(\min _{\omega \in \mathbf{R}_{0+}} \operatorname{Re} G(\mathbf{i} \omega)\right)\left\langle u_{t}, u_{t}\right\rangle+\int_{0}^{t} c\left(\tau, x_{0}\right) u(\tau) d \tau \\
& =\left(\min _{\omega \in \mathbf{R}_{0+}} \operatorname{Re} G(\mathbf{i} \omega)\right)\langle u, u\rangle_{t} \\
& +\int_{0}^{t} c\left(\tau, x_{0}\right) u(\tau) d \tau ; \quad \forall t \in \mathbf{R}_{0+},
\end{aligned}
$$

where $F(\mathbf{i} \omega)$ is the pointwise value at frequency $\omega$ of $F$ : $\mathbf{C} \rightarrow \mathbf{C}$, the Fourier transform of $f: \mathbf{R} \rightarrow \mathbf{R}$ provided that it exists with $\mathbf{i}=\sqrt{-1}$ being the complex unit. Note that, in the previous expressions, the integral expressions have been also denoted by inner products $\langle\cdot, \cdot\rangle_{t} \equiv\left\langle{ }_{t}, \cdot\right\rangle \equiv$ $\left\langle\cdot,{ }_{t}\right\rangle$ on the time interval $[0, t]$ for the given $t \in \mathbf{R}_{0+}$, all of them being equivalent to inner products of truncated functions for the given $t \in \mathbf{R}_{0+}$ on the Hilbert space $L^{2}[0, \infty)$. Equivalently, integrals of complex Fourier transforms on the whole imaginary axis are got through Parseval's theorem and denoted by $\left\langle G U_{t}, U_{t}\right\rangle$ involving the impulse response (i.e., the transfer function evaluated on the imaginary complex axis) of the system and the Fourier transform of the truncated input. Now, assume that the controller is

$$
u(t)=k(t) r(t)-\varphi(t, y(t)) ; \quad \forall t \in \mathbf{R}_{0+} .
$$

$r: \mathbf{R} \rightarrow \mathbf{R}$ is an exogenous reference signal which is piecewise continuous on $\mathbf{R}_{0+}$, and $\varphi:[0, t] \times \mathbf{R} \rightarrow \mathbf{R}$ is any piecewise continuous nonlinear time-varying function which satisfies the following integral-type constraint:

$$
\int_{0}^{t} \varphi(\tau, y(\tau)) y(\tau) d \tau \geq-\gamma\left(t, x_{0}\right), \quad \forall t \in \mathbf{R}_{0+}
$$

then

$$
\begin{aligned}
E(t) & =\int_{0}^{t} y(\tau) u(\tau) d \tau \\
& =\int_{0}^{t} y(\tau) k(\tau) r(\tau) d \tau-\int_{0}^{t} y(\tau) \varphi(\tau, y(\tau)) d \tau \\
& \leq \gamma\left(t, x_{0}\right)+\int_{0}^{t} y(\tau) k(\tau) r(\tau) d \tau .
\end{aligned}
$$

Note that any hodograph $G(\mathbf{i} \omega)$ has the symmetry rules $\operatorname{Re} G(\mathbf{i} \omega)=\operatorname{Re} G(-\mathbf{i} \omega)$ and $\operatorname{Im} G(\mathbf{i} \omega)=-\operatorname{Im} G(-\mathbf{i} \omega)$. Also, $\mu(G) \geq \min _{\omega \in \mathbf{R}_{0+}} \operatorname{Re} G(\mathbf{i} \omega)>0$. Thus, one gets by combining (37) and (40)

$$
\begin{gathered}
\left(\min _{\omega \in \mathbf{R}_{0+}} \operatorname{Re} G(\mathbf{i} \omega)\right) \int_{0}^{t}|u(\tau)|^{2} d \tau+\int_{0}^{t} c\left(\tau, x_{0}\right) u(\tau) d \tau \\
\leq \gamma\left(t, x_{0}\right)+\int_{0}^{t} y(\tau) k(\tau) r(\tau) d \tau ; \quad \forall t \in \mathbf{R}_{0+} .
\end{gathered}
$$

Decompose $[0, t]=I_{1 \varepsilon}(t) \cup I_{2 \varepsilon}(t)$ for each $t \in \mathbf{R}_{0+}$, where

$$
\begin{aligned}
& I_{1 \varepsilon}(t)=\{\tau \in[0, t]:|u(\tau)| \leq \varepsilon\}, \\
& I_{2 \varepsilon}(t)=\{\tau \in[0, t]:|u(\tau)|>\varepsilon\},
\end{aligned}
$$


for some given prefixed $\varepsilon \in \mathbf{R}_{+}$. Note that one (but not both) of the disjoint sets $I_{i \varepsilon}(t)$ for $i=1,2$ can be empty. Then, by direct calculations one gets the following:

$$
\begin{aligned}
& \left(\min _{\omega \in \mathbf{R}_{0+}} \operatorname{Re} G(\mathbf{i} \omega)\right) \int_{0}^{t}|u(\tau)|^{2} d \tau+\int_{0}^{t} c\left(\tau, x_{0}\right) u(\tau) d \tau \\
& \geq\left(\min _{\omega \in \mathbf{R}_{0+}} \operatorname{Re} G(\mathbf{i} \omega)\right) \int_{0}^{t}|u(\tau)|^{2} d \tau-\varepsilon \int_{I_{1}(t)}\left|c\left(\tau, x_{0}\right)\right| d \tau \\
& \quad+\int_{I_{2}(t)} \frac{c\left(\tau, x_{0}\right)}{u(\tau)}|u(\tau)|^{2} d \tau \\
& \geq\left(\min _{\omega \in \mathbf{R}_{0+}} \operatorname{Re} G(\mathbf{i} \omega)\right) \int_{0}^{t}|u(\tau)|^{2} d \tau-\varepsilon \int_{I_{1}(t)}\left|c\left(\tau, x_{0}\right)\right| d \tau \\
& \quad-\left.\int_{I_{2}(t)}\left|\frac{c\left(\tau, x_{0}\right)}{u(\tau)}\right| u(\tau)\right|^{2} d \tau \\
& \geq\left(\min _{\omega \in \mathbf{R}_{0+}} \operatorname{Re} G(\mathbf{i} \omega)-\varepsilon^{-1} \max _{t \in \mathbf{R}_{0+}}\left|c\left(t, x_{0}\right)\right|\right) \\
& \quad \times \int_{0}^{t}|u(\tau)|^{2} d \tau-\varepsilon \int_{I_{1}(t)}\left|c\left(\tau, x_{0}\right)\right| d \tau ; \quad \forall t \in \mathbf{R}_{0+} .
\end{aligned}
$$

Assume that $\sup _{t \in \mathbf{R}_{0+}}|k(t)| \leq \bar{k}$ and $\sup _{t \in \mathbf{R}_{0+}}|r(t)| \leq \bar{r}$. Then, one gets from (41) and (43) that

$$
\begin{aligned}
\int_{0}^{t}\left(|u(\tau)|-\bar{k} \bar{r} \max _{\omega \in \mathbf{R}_{0+}}|G(\mathbf{i} \omega)|\right)|u(\tau)| d \tau \\
\leq \frac{1}{\min _{\omega \in \mathbf{R}_{0+}} \operatorname{Re} G(\mathbf{i} \omega)-\varepsilon^{-1} \max _{t \in \mathbf{R}_{0+}}\left|c\left(t, x_{0}\right)\right|} \\
\times\left(\gamma\left(t, x_{0}\right)+\varepsilon \int_{I_{1}(t)}\left|c\left(\tau, x_{0}\right)\right| d \tau\right), \\
\forall t \in \mathbf{R}_{0+} .
\end{aligned}
$$

This relation leads to the following result.

Proposition 11. Assume that

(1) $k, r \in L_{\infty}\left(\mathbf{R}_{0+}\right)$ so that $\sup _{t \in \mathbf{R}_{0+}}|k(t)| \leq \bar{k}<\infty$ and $\sup _{t \in \mathbf{R}_{0+}}|r(t)| \leq \bar{r}<\infty$,

(2) the transfer function $G(s)$ is strongly strictly positive real; that is, $\operatorname{Re} G(s)>0$ for all complex $s$ with $\operatorname{Re} s \geq 0$,

(3) $+\infty>\limsup _{t \rightarrow \infty}\left(\gamma\left(t, x_{0}\right)\right) \geq \liminf _{t \rightarrow \infty}(\gamma(t$, $\left.\left.x_{0}\right)\right)>0 ; \forall x_{0} \in \mathbf{R}^{n}$.

Then, one gets the following properties for any given initial state $x_{0} \in \mathbf{R}^{n}$.

(i) $u, y \in L_{\infty}\left(\mathbf{R}_{0+}\right)$.

(ii) $\liminf \operatorname{in}_{t \rightarrow \infty} \int_{0}^{t} y(\tau) k(\tau) r(\tau) d \tau>-\infty$; $\liminf _{t \rightarrow \infty}$ $\left(u^{2}(t)-y(t) k(t) r(t)\right) \leq 0$.

(iii) If $|u(t)|^{2} \geq y(t) k(t) r(t) ; \forall t \in \mathbf{R}_{0+}$, then $\exists \lim _{t \rightarrow \infty}\left(|u(t)|^{2}-y(t) k(t) r(t)\right)=0$. If, in addition, $k(t)=k$ and $r(t)=r$ are nonzero constants; $\forall t \in$ $\mathbf{R}_{0+}$, then $\exists \lim _{t \rightarrow \infty} y(t)=y_{\infty}$ and $\exists \lim _{t \rightarrow \infty} u(t)=$ $u_{\infty}=k r \int_{0}^{\infty} g(\tau) d \tau$ and $\exists \lim _{t \rightarrow \infty} y(t)=y_{\infty}=$ $k r\left(\int_{0}^{\infty} g(\tau) d \tau\right)^{2}$.

(iv) if $r \equiv 0$, then $u(t) \rightarrow 0$ and $y(t) \rightarrow 0$ as $t \rightarrow \infty$ and are both square-integrable on $\mathbf{R}_{0+} ; \forall x_{0} \in \mathbf{R}^{n}$. Thus, the closed-loop dynamic system (36), (38) is asymptotically hyperstable (i.e., globally asymptotically Lyapunov's stable, [1-3]) since the state of any minimal state-space realization is also square-integrable on $\mathbf{R}_{0+}$, and it converges asymptotically to zero as time tends to infinity for any controller device $\varphi:[0, t] \times \mathbf{R} \rightarrow \mathbf{R}$ satisfying (39).

Proof. Since the transfer function $G(s)$ is strictly positive real then it is strictly stable (i.e. all its poles are in $\operatorname{Re} s \leq-\rho<0$ for some $\rho \in \mathbf{R}_{+}$) and $\operatorname{Re} G(s)>0$ for all complex $s$ with $\operatorname{Re} s \geq 0$. Since it is, furthermore, strongly positive real (i.e., a strictly positive operator on $\left.L^{2}\left(\mathbf{R}_{0+}\right)\right)$, and it is associated to a dynamic system, so that it is realizable, then it is rational with pole-zero excess is zero (otherwise, if the pole-zero excess was +1 , then it could not be strongly strictly positive real since $\lim _{\omega \rightarrow \pm \infty} \operatorname{Re} G(\mathbf{i} \omega)=0$, and if the pole-zero excess was -1 then it would not be realizable.) Since it has the same number of zeros and poles, and it is strongly strictly positive real, then its modulus is everywhere bounded in its definition domain, invertible, and of bounded inverse, so that one has

$$
0<\min _{\omega \in \mathbf{R}_{0+}} \operatorname{Re} G(\mathbf{i} \omega) \leq \max _{\omega \in \mathbf{R}_{0+}}|G(\mathbf{i} \omega)|<+\infty .
$$

Note that $\int_{I_{1}(t)}\left|c\left(\tau, x_{0}\right)\right| d \tau \leq \int_{0}^{\infty}\left|c\left(\tau, x_{0}\right)\right| d \tau=v\left(x_{0}\right)<$ $+\infty$ since $\lim _{t \rightarrow \infty}\left|c\left(t, x_{0}\right)\right|=0$ at exponential rate since the dynamic system is strictly stable. Since $\varepsilon \in \mathbf{R}_{+}$can be chosen arbitrarily to build the disjoint union $I_{1 \varepsilon}(t) \cup$ $I_{2 \varepsilon}(t)$ equalizing $[0, t] ; \forall t \in \mathbf{R}_{0+}$, then choose $\varepsilon>$ $\left|c\left(t, x_{0}\right)\right| /\left(\min _{\omega \in \mathbf{R}_{0+}} \operatorname{Re} G(\mathbf{i} \omega)\right)$. Now, assume that $u: \mathbf{R} \rightarrow \mathbf{R}$ is unbounded. Since, it is piecewise continuous with eventual bounded discontinuities, then $\lim _{t \rightarrow \infty} \int_{0}^{t}|u(\tau)| d \tau=\infty$ which implies that $\int_{0}^{t}\left(|u(\tau)|-\bar{k} \bar{r} \max _{\omega \in \mathbf{R}_{0+}}|G(\mathbf{i} \omega)|\right)|u(\tau)| d \tau$ is strictly increasing so that the subsequent contradiction follows

$$
\begin{aligned}
&+\infty= \lim _{t \rightarrow \infty} \int_{0}^{t}\left(|u(\tau)|-\bar{k} \bar{r} \max _{\omega \in \mathbf{R}_{0+}}|G(\mathbf{i} \omega)|\right)|u(\tau)| d \tau \\
& \leq \lim _{t \rightarrow \infty} \int_{0}^{t}\left(|u(\tau)|^{2}-k(\tau) r(t) y(\tau)\right) d \tau \\
& \leq \frac{1}{\min _{\omega \in \mathbf{R}_{0+}} \operatorname{Re} G(\mathbf{i} \omega)-\varepsilon^{-1} \max _{t \in \mathbf{R}_{0+}}\left|c\left(t, x_{0}\right)\right|} \\
& \times\left(\gamma\left(t, x_{0}\right)+\varepsilon \int_{I_{1}(t)}\left|c\left(\tau, x_{0}\right)\right| d \tau\right)<+\infty \\
& \forall t \in \mathbf{R}_{0+} .
\end{aligned}
$$

Thus, $u \in L_{\infty}\left(\mathbf{R}_{0+}\right)$. Since $G(s)$ is strictly stable and $u \in L_{\infty}\left(\mathbf{R}_{0+}\right)$, then $y \in L_{\infty}\left(\mathbf{R}_{0+}\right)$. Property (i) has 
been proved. On the other hand, if $\liminf _{t \rightarrow \infty}\left(|u(t)|^{2}-\right.$ $y(t) k(t) r(t))>0$, then $\lim _{t \rightarrow \infty} \int_{0}^{t}\left(|u(\tau)|^{2}-k(\tau) r(t) y(\tau)\right) d \tau=$ $+\infty$, and the above contradiction holds. Then, $\liminf _{t \rightarrow \infty}\left(|u(t)|^{2}-y(t) k(t) r(t)\right) \leq 0$. Note also that if $\lim _{t \rightarrow \infty} \int_{0}^{t} y(\tau) k(\tau) r(\tau) d \tau=-\infty$, then the subsequent contradiction follows

$$
\begin{aligned}
0 \leq & \lim _{t \rightarrow \infty} \int_{0}^{t}|u(\tau)|^{2} d \tau \\
\leq & \frac{1}{\min _{\omega \in \mathbf{R}_{0+}} \operatorname{Re} G(\mathbf{i} \omega)-\varepsilon^{-1} \max _{t \in \mathbf{R}_{0+}}\left|c\left(t, x_{0}\right)\right|} \\
& \times\left(\gamma\left(t, x_{0}\right)+\int_{0}^{t}\left[\varepsilon\left|c\left(\tau, x_{0}\right)\right|+k(\tau) r(t) y(\tau)\right] d \tau\right) \\
= & -\infty, \quad \forall t \in \mathbf{R}_{0+} .
\end{aligned}
$$

Then, $\liminf _{t \rightarrow \infty} \int_{0}^{t} y(\tau) k(\tau) r(\tau) d \tau>-\infty$. Property (ii) has been proven.

Note that $\exists \lim _{t \rightarrow \infty}\left(|u(t)|^{2}-y(t) k(t) r(t)\right)=0$ if $|u(t)|^{2} \geq y(t) k(t) r(t) ; \forall t \in \mathbf{R}_{0+}$ is a direct consequence of $\liminf _{t \rightarrow \infty}\left(|u(t)|^{2}-y(t) k(t) r(t)\right) \leq 0$ from Property (ii). This proves the first part of Property (iii). Also, if $k(t)=$ $k$ and $r(t)=r$ are nonzero constants; $\forall t \in \mathbf{R}_{0+}$, then $\lim _{t \rightarrow \infty}\left(|u(t)|^{2}-y(t) k r\right)=0$.

Now, if $r(t)$ is identically zero in $\mathbf{R}_{0+}$, then

$$
\begin{aligned}
\lim _{t \rightarrow \infty} \int_{0}^{t}|u(\tau)|^{2} d \tau & \\
\leq & \frac{1}{\min _{\omega \in \mathbf{R}_{0+}} \operatorname{Re} G(\mathbf{i} \omega)-\varepsilon^{-1} \max _{t \in \mathbf{R}_{0+}}\left|c\left(t, x_{0}\right)\right|} \\
& \times\left(\gamma\left(t, x_{0}\right)+\varepsilon \int_{I_{1}(t)}\left|c\left(\tau, x_{0}\right)\right| d \tau\right)<+\infty, \\
\forall t & \in \mathbf{R}_{0+}
\end{aligned}
$$

leads to $\lim _{t \rightarrow \infty} u(t)=0$ exponentially and the $\lim _{t \rightarrow \infty}$ $y(t)=0 ; \forall x_{0} \in \mathbf{R}^{n}$ since $G(s)$ is strongly strictly positive real so that the internal state of any minimal state-space realization is uniformly bounded, and it converges asymptotically to zero as time tends to infinity. Thus, asymptotic hyperstability follows for any $\varphi:[0, t] \times \mathbf{R} \rightarrow \mathbf{R}$ satisfying (38). As a result, Property (iv) has been proven.

Note that the property of asymptotic hyperstability is independent of each particular controller provided that it belongs to a class that satisfies the integral relation (39) for some positive finite real $\gamma$. The particular case when the nonlinear controller is nonlinear, but time-invariant, while satisfying the corresponding integral constraint (39), is said to be the Popov-type absolute stability problem implying closed-loop global asymptotic Lyapunov's stability. If the input-output euclidean inner product (associated with instantaneous power) under the integral symbol, rather than the inner product on the Hilbert space (associated with the energy), satisfies a parallel inequality, then the problem is said to be that of the Lure's absolute stability problem [4-8]. It is, therefore, useful to describe the global asymptotic stability of classes of closed-loop systems of the given form under certain tolerated components dispersions. Proposition 11 also implies directly that any nonminimal state-space realization associated with strictly stable zero-pole cancellations of the transfer function is globally asymptotically Lyapunov stable. This follows since the transfer function remains invariant under zero-pole cancellations, so it is identical to that of the minimum state space realization, so that the operator is kept strictly positive and invertible although either controllability or observability (or both) becomes lost [29-31]. A generalization of the previous result to the study of hyperstability of composite connections [32] as well to Ulman-type extended stability $[33,34]$ of continuous-time dynamic systems can be performed based on the study given in [32].

The subsequent example is a discrete version of the previous one.

Example 2. Example 1 has a direct parallel discrete-time counterpart as discussed in the sequel. Define the truncated sequence on $[0, k T] ; \forall k \in \Gamma=\mathbf{Z}_{0+}$ of the real sequence $\left\{f_{k} \equiv f(k T)\right\}_{k \in \mathbf{Z}_{0+}}$ as follows:

$$
f_{j}(k)=P_{j} f(k T)= \begin{cases}f_{j} \equiv f(j T) & \text { for } j \in[0, k] \cap \mathbf{Z} \\ 0 & \text { otherwise; }\end{cases}
$$

$$
\forall k \in \mathbf{Z}_{0+} \text { ， }
$$

where $T>0$ is the sampling period. Thus, the output of a single-input single-output linear continuous-time dynamic system of $n$th order and initial state $x(0)=x_{0} \in \mathbf{R}^{n}$ under a piecewise continuous control with eventual isolated bounded discontinuities $u: \mathbf{R} \rightarrow \mathbf{R} \cap H$, now the Hilbert space being $H=\ell^{2}\left(\mathbf{Z}_{0+}\right)$, is

$$
\begin{aligned}
y_{k} & =\sum_{j=0}^{k} g^{d}(k, j) u_{j}+c\left(k, x_{0}\right) \\
& =\sum_{j=-\infty}^{\infty} g^{d}(k, j) u_{k}(j)+c_{k}\left(x_{0}\right) \\
& =\left(g^{d} \circ u\right)(k)+c_{k}\left(x_{0}\right), \quad \forall k \in \mathbf{Z}_{0+},
\end{aligned}
$$

where $\mathbf{Z}_{0+}=\{z \in \mathbf{Z}: z \geq 0\}$, “o" stands for the discrete convolution operator, $c_{k}=c_{k}\left(x_{0}\right) \equiv c_{k}\left(k T, x_{0}\right)$ and $\left\{g^{d}(k, j)\right\}_{k, j \in \mathbf{Z}_{0+}}$ is the impulse response sequence since the dynamic system is realizable $g^{d}(k, j)=0$ for $j>k$. If this dynamic system is the same system as in the previous example subject to a piecewise control sequence $\left\{u_{k}\right\}_{k \in \mathbf{Z}_{0+}}$, with $u_{k}=$ $u(k T) ; \forall k \in \mathbf{Z}_{0+}$, then $g^{d}(k, 0)=\left(1-q^{-1}\right) \mathbf{L}^{-1}(G(s) / s)_{t=k T}$; $\forall k \in \mathbf{Z}_{0+}$ where $q^{-1}$ is the one-step delay operator such that $f_{k}=q^{-1} f_{k+1}$. In this case, the discrete controller is

$$
u_{k}=k_{k} r_{k}-\varphi_{k}\left(k, y_{k}\right) ; \quad \forall k \in \mathbf{Z}_{0+} .
$$


$r: \mathbf{R} \rightarrow \mathbf{R}$ is an exogenous reference sequence, and $\varphi:$ $[0, k] \times \mathbf{R} \rightarrow \mathbf{R}$ are the elements of any nonlinear timevarying real sequence $\left\{\varphi_{k}=\varphi_{k}\left(k, y_{k}\right)\right\}_{k \in \mathbf{Z}_{0+}}$ which satisfies the following summation-type constraint:

$$
\sum_{j=0}^{k} \varphi_{j}\left(j, y_{j}\right) y_{j} \geq-\gamma_{k}\left(x_{0}\right) ; \quad \forall k \in \mathbf{Z}_{0+} .
$$

A close discussion to that of the former example by using the discrete Parseval theorem and inner products on the Hilbert space of square-summable sequences $\ell^{2}\left(\mathbf{Z}_{0+}\right)$ yields

$$
\begin{aligned}
& E_{k}=\sum_{j=0}^{k} y_{j} u_{j}=\sum_{j=-\infty}^{\infty} y_{j} u_{j}(k) \\
& =\frac{1}{2 \pi} \oint_{|z|=1} z^{-1} Y(z) U\left(k, z^{-1}\right) d z \\
& =\frac{1}{2 \pi} \int_{0}^{2 \pi} Y\left(e^{\mathbf{i} \varphi}\right) U\left(k, e^{-\mathbf{i} \varphi}\right) e^{-\mathbf{i} \varphi} d \varphi \\
& =\langle y, u(k)\rangle=\frac{1}{2 \pi}\langle Y, U(k)\rangle \\
& =\frac{1}{2 \pi}\left\langle G^{d} U(k), U(k)\right\rangle+\sum_{j=0}^{k} c_{j}\left(x_{0}\right) u_{j} \\
& =\frac{1}{2 \pi}\left\langle G^{d},|U(k)|^{2}\right\rangle+\sum_{j=0}^{k} c_{j}\left(x_{0}\right) u_{j} \\
& \geq \frac{1}{2 \pi}\left(\min _{\varphi \in[0,2 \pi]} \operatorname{Re} G^{d}\left(e^{\mathbf{i} \varphi}\right)\right) \int_{0}^{2 \pi}\left|U\left(k, e^{\mathbf{i} \varphi}\right)\right|^{2} d \varphi \\
& =\left(\min _{\varphi \in[0,2 \pi]} \operatorname{Re} G^{d}\left(e^{\mathbf{i} \varphi}\right)\right)\left(\sum_{j=-\infty}^{\infty} u_{j}^{2}(k)\right)+\sum_{j=0}^{k} c_{j}\left(x_{0}\right) u_{j} \\
& =\left(\min _{\varphi \in[0,2 \pi]} \operatorname{Re} G^{d}\left(e^{\mathbf{i} \varphi}\right)\right)\left(\sum_{j=0}^{k} u_{j}^{2}(k)\right) \\
& +\sum_{j=0}^{k} c_{j}\left(x_{0}\right) u_{j}, \quad \forall k \in \mathbf{Z}_{0+} .
\end{aligned}
$$

By using (53), one gets a discrete counterpart of (44) as follows:

$$
\begin{aligned}
& \sum_{j=0}^{k}\left(\left|u_{j}\right|-\bar{k} \bar{r} \max _{\varphi \in[0,2 \pi]}\left|G^{d}\left(e^{\mathbf{i} \varphi}\right)\right|\right)\left|u_{j}\right| \\
& \leq \frac{1}{\min _{\varphi \in[0,2 \pi]} \operatorname{Re} G^{d}\left(e^{\mathbf{i} \varphi}\right)-\varepsilon^{-1} \max _{k \in \mathbf{Z}_{0+}}\left|c_{k}\left(x_{0}\right)\right|} \\
& \times\left(\gamma_{k}\left(x_{0}\right)+\varepsilon \sum_{j=0}^{k}\left|c_{k}\left(x_{0}\right)\right|\right), \quad \forall k \in \mathbf{Z}_{0+}
\end{aligned}
$$

which leads to the subsequent result which is the discretetime counterpart of Proposition 11, whose proof is close to that of Proposition 11, and it is then omitted.
Proposition 12. Assume that

(1) $k, r \in \ell_{\infty}\left(\mathbf{Z}_{0+}\right)$ so that $\sup _{j \in \mathbf{Z}_{0+}}\left|k_{j}\right| \leq \bar{k}<\infty$ and $\sup _{k \in \mathbf{Z}_{0+}}\left|r_{k}\right| \leq \bar{r}<\infty$,

(2) the discrete function $G^{d}(z)$ is strongly strictly positive real; that is, $\operatorname{Re} G(z)>0$ for all complex $z$ with $|z| \geq 1$.

(3) $+\infty>\lim \sup _{k \rightarrow \infty}\left(\gamma_{k}\left(x_{0}\right)\right) \geq \liminf _{t \rightarrow \infty}\left(\gamma_{k}\left(x_{0}\right)\right)>$ $0 ; \forall x_{0} \in \mathbf{R}^{n}$.

Then, one gets the following properties for any given initial state $x_{0} \in \mathbf{R}^{n}$.

(i) $u, y \in \ell_{\infty}\left(\mathbf{Z}_{0+}\right)$.

(ii) $\liminf \operatorname{int\infty }_{k \rightarrow \infty}\left(\sum_{j=0}^{k} y_{j} k_{j} r_{j}\right)>-\infty ; \liminf _{j \rightarrow \infty}\left(u_{j}^{2}-\right.$ $\left.y_{j} k_{j} r_{j}\right) \leq 0$.

(iii) If $u_{j}^{2} \geq y_{j} k_{j} r_{j} ; \forall j \in \mathbf{Z}_{0+}$, then $\exists \lim _{j \rightarrow \infty}\left(u_{j}^{2}-y_{j} k_{j} r_{j}\right)=$ 0. If, in addition, $k_{j}=k$ and $r_{j}=r$ are nonzero constants; $j \in \mathbf{Z}_{0+}$, then $\exists \lim _{j \rightarrow \infty} y_{j}=y_{\infty}$ and $\exists \lim _{j \rightarrow \infty} u_{j}=u_{\infty}=k r\left(\sum_{j=0}^{\infty} g_{j}\right)$ and $\exists \lim _{j \rightarrow \infty} y_{j}=$ $y_{\infty}=k r\left(\sum_{j=0}^{\infty} g_{j}\right)^{2}$.

(iv) If $r \equiv 0$, then $u_{j} \rightarrow 0$ and $y_{j} \rightarrow 0$ as $j \rightarrow \infty$, and they are both square-summable on $\mathbf{Z}_{0+} ; \forall x_{0} \in \mathbf{R}^{n}$. Thus, the closed-loop discrete dynamic system (50)-(51) is asymptotically hyperstable for any controller device of output sequence $\left\{\varphi_{k}=\varphi_{k}\left(k, y_{k}\right)\right\}_{k \in \mathbf{Z}_{0+}}$ satisfying the discrete summation inequality $\sum_{j=0}^{k} \varphi_{j}\left(j, y_{j}\right) y_{j} \geq$ $-\gamma_{k}\left(x_{0}\right) ; \forall k \in \mathbf{Z}_{0+}$

The following example links asymptotic hyperstability of a discrete dynamic system with a unique equilibrium point which is also a fixed point.

Example 3. Assume that, in Example 2, a feedback stabilizing discrete control law $u_{t}=-\varphi_{t}\left(t, y_{t-1}\right) y_{t-1}$ satisfying the constraint $\sum_{j=0}^{t} \varphi_{j}\left(j, y_{j}\right) y_{j} \geq-\gamma_{t}\left(x_{0}\right) \geq-\gamma>\infty ; \forall j, t \in \mathbf{Z}_{0+}$ is injected to the system (1), neglecting initial conditions, and equivalently if the initial conditions are zero (this assumption does not affect the stability study,) we get

$$
\begin{aligned}
P_{t} y & =y_{t}=-\left(P_{t} G\right) u=-\left(P_{t} G \varphi_{t}\left(t, y_{t-1}\right) P_{t-1}\right) y \\
& =-\left(P_{t} G \varphi_{t}\left(t, P_{t-1} y\right) P_{t-1}\right) y ; \quad \forall t \in \mathbf{Z}_{0+}
\end{aligned}
$$

so that the closed-loop system can be described by the operator $Q: H_{e}\left|\ell^{2}\left(\mathbf{Z}_{0+}\right) \rightarrow H_{e}\right| \ell^{2}\left(\mathbf{Z}_{0+}\right)$ represented as

$$
y_{t}=P_{t} Q P_{t-1} y=-P_{t} G \varphi_{t}\left(t, y_{t-1}\right) y_{t-1} ; \quad \forall t \in \mathbf{Z}_{0+}
$$

or, equivalently, as

$$
P_{t}\left(I+G \varphi_{t}\left(t, P_{t-1} y\right) P_{t-1}\right) y=0 ; \quad \forall t \in \mathbf{Z}_{0+} .
$$

Assume that $Q: \ell^{2}[0, z) \rightarrow \ell^{2}[0, z)$ for any $z \in \mathbf{Z}_{+}$ is stable, positive, one-to-one, and of closed range. Then, $Q: H_{e}\left|\ell^{2}\left(\mathbf{Z}_{0+}\right) \rightarrow H_{e}\right| \ell^{2}\left(\mathbf{Z}_{0+}\right)$, where $H_{e}=\bigcup_{z \in \mathbf{Z}_{+}} \ell^{2}[0, z)$ 
is positive, bounded and of closed range, invertible and of nonzero minimum modulus; and

$$
0 \leq E(t)=\left\langle P_{t} Q P_{t-1} y, P_{t} y\right\rangle \leq \gamma<\infty ; \quad \forall t \in \mathbf{Z}_{0+} .
$$

Since $E(t)$ is nonnegative, bounded, and nondecreasing, $y_{t} \rightarrow 0$ as $t\left(\in \mathbf{Z}_{0+}\right) \rightarrow \infty$, and then $u_{t}=-\varphi_{t}\left(t, y_{t-1}\right) y_{t-1} \rightarrow$ 0 as $t\left(\in \mathbf{Z}_{0+}\right) \rightarrow \infty$. One gets for any given finite integer $T>$ 0 that $\lim _{\Gamma \ni t \rightarrow \infty}\left(P_{t+T} u-P_{r} u\right)=\lim _{\Gamma \ni t \rightarrow \infty}\left(P_{t+T} y-P_{t} y\right)=0$. Thus, $\bar{y}(T) \equiv 0\left(\in \ell^{2}[t, t+T]\right)$ is the unique fixed point of $Q: H_{e}\left|\ell^{2}[t, t+T] \rightarrow H_{e}\right| \ell^{2}[t+T, t+2 T] ; \forall t \in \Gamma$.

\section{Acknowledgments}

The author is very grateful to the Spanish Government for its support of this research through Grant DPI2012-30651 and to the Basque Government for its support of this research through Grants IT378-10 and SAIOTEK S-PE12UN015. The author is also grateful to the University of the Basque Country for its financial support through Grant UFI 2011/07.

\section{References}

[1] V.-M. Popov, Hyperstability of Control Systems, Springer, New York, NY, USA, 1973.

[2] M. de la Sen, "A result on the hyperstability of a class of hybrid dynamic systems," IEEE Transactions on Automatic Control, vol. 42, no. 9, pp. 1335-1339, 1997.

[3] M. de la Sen, "Preserving positive realness through discretization," Positivity, vol. 6, no. 1, pp. 31-45, 2002.

[4] B. Xu, X. Liu, and X. Liao, "Absolute stability of Lurie systems with impulsive effects," Computers \& Mathematics with Applications, vol. 47, no. 2-3, pp. 419-425, 2004.

[5] B. Zhou, G.-R. Duan, and J. Lam, "On the absolute stability approach to quantized feedback control," Automatica, vol. 46, no. 2, pp. 337-346, 2010.

[6] A. Kazemy and M. Farrokhi, "Robust absolute stability analysis of multiple time-delay Lur'e systems with parametric uncertainties," Asian Journal of Control, vol. 15, no. 1, pp. 203-213, 2013.

[7] D. Wang and F. Liao, "Absolute stability of Lurie direct control systems with time-varying coefficients and multiple nonlinearities," Applied Mathematics and Computation, vol. 219, no. 9, pp. 4465-4473, 2013.

[8] H.-B. Zeng, Y. He, M. Wu, and S.-P. Xiao, "Absolute stability and stabilization for Lurie networked control systems," International Journal of Robust and Nonlinear Control, vol. 21, no. 14, pp. 16671676, 2011.

[9] L.-J. Lin, Z.-T. Yu, and C.-S. Chuang, "Weak and strong convergence theorems for asymptotically pseudo-contraction mappings in the intermediate sense in Hilbert spaces," Journal of Global Optimization, vol. 56, no. 1, pp. 165-183, 2013.

[10] X. Chai and Y. Song, "Convergence theorem for an iterative algorithm of lamda-strict pseudocontractions," Fixed Point Theory and Applications, vol. 2011, article 95, 2011.

[11] K. Xiong, "Necessary and sufficient conditions for the existence of a Lyapunov function with 'a quadratic form plus an integral term,' International Journal of Control, vol. 64, no. 4, pp. 707719, 1996.

[12] Y. Yao, H. Zhou, and Y.-C. Liou, "Weak and strong convergence theorems for an asymptotically $k$-strict pseudo-contraction and a mixed equilibrium problem," Journal of the Korean Mathematical Society, vol. 46, no. 3, pp. 561-576, 2009.

[13] P. Duan, "Convergence theorems concerning hybrid methods for strict pseudocontractions and systems of equilibrium problems," Journal of Inequalities and Applications, vol. 2010, Article ID 396080, 14 pages, 2010.

[14] Y. H. Yao and R. D. Chen, "Strong convergence theorems for strict pseudo-contractions in Hilbert spaces," Journal of Applied Mathematics and Computing, vol. 32, no. 1, pp. 69-82, 2010.

[15] W. Shatanawi and M. Postolache, "Common fixed point results of mappings for nonlinear contraction of cyclic form in ordered metric spaces," Fixed Point Theory and Applications, vol. 2013, artcile 60, 2013.

[16] N. Hussain and H. K. Pathak, "Common fixed point and approximation results for $H$-operator pair with applications," Applied Mathematics and Computation, vol. 218, no. 22, pp. 11217-11225, 2012.

[17] H. K. Nashine and M. S. Khan, "An application of fixed point theorem to best approximation in locally convex space," Applied Mathematics Letters, vol. 23, no. 2, pp. 121-127, 2010.

[18] A. Latif and M. A. Kutbi, "Fixed points for $w$-contractive multimaps," International Journal of Mathematics and Mathematical Sciences, vol. 2009, Article ID 769467, 7 pages, 2009.

[19] T. Husain and A. Latif, "Fixed points of multivalued nonexpansive maps," International Journal of Mathematics and Mathematical Sciences, vol. 14, no. 3, pp. 421-430, 1991.

[20] M. S. Khan, "Common fixed point theorems for multivalued mappings," Pacific Journal of Mathematics, vol. 95, no. 2, pp. 337347, 1981.

[21] B. Samet, C. Vetro, and P. Vetro, "Fixed point theorems for $\alpha-\psi$-contractive type mappings," Nonlinear Analysis: Theory, Methods \& Applications, vol. 75, no. 4, pp. 2154-2165, 2012.

[22] M. I. Berenguer, M. A. Fortes, A. I. Garralda Guillem, and M. Ruiz Galán, "Linear Volterra integro-differential equation and Schauder bases," Applied Mathematics and Computation, vol. 159, no. 2, pp. 495-507, 2004.

[23] D. Gámez, A. I. Garralda Guillem, and M. Ruiz Galán, "Highorder nonlinear initial-value problems countably determined," Journal of Computational and Applied Mathematics, vol. 228, no. 1, pp. 77-82, 2009.

[24] W.-S. Du, "New cone fixed point theorems for nonlinear multivalued maps with their applications," Applied Mathematics Letters, vol. 24, no. 2, pp. 172-178, 2011.

[25] M. de la Sen, "About robust stability of dynamic systems with time delays through fixed point theory," Fixed Point Theory and Applications, vol. 2008, Article ID 480187, 20 pages, 2008.

[26] M. de la Sen, "About robust stability of Caputo linear fractional dynamic systems with time delays through fixed point theory," Fixed Point Theory and Applications, vol. 2011, Article ID 867932, 19 pages, 2011.

[27] M. de la Sen, "Total stability properties based on fixed point theory for a class of hybrid dynamic systems," Fixed Point Theory and Applications, vol. 2009, Article ID 826438, 19 pages, 2009.

[28] A. Feintuch, Robust Control Theory in Hilbert Space, vol. 130 of Applied Mathematical Sciences, Springer, New York, NY, USA, 1998.

[29] V. Ionescu, C. Oară, and M. Weiss, Generalized Riccati Theory and Robust Control. A Popov Function Approach, John Wiley \& Sons, New York, NY, USA, 1999. 
[30] C. Bianca, M. Ferrara, and L. Guerrini, "The Cai model with time delay: existence of periodic solutions and asymptotic analysis," Applied Mathematics \& Information Sciences, vol. 7, no. 1, pp. 21-27, 2013.

[31] C. Udrişte, M. Ferrara, D. Zugrăvescu, and F. Munteanu, "Controllability of a nonholonomic macroeconomic system," Journal of Optimization Theory and Applications, vol. 154, no. 3, pp. 1036-1054, 2012.

[32] M. de la Sen, "Stability of composite systems with an asymptotically hyperstable subsystem," International Journal of Control, vol. 44, no. 6, pp. 1769-1775, 1986.

[33] N. Brillouët-Belluot, J. Brzdęk, and K. Ciepliński, "On some recent developments in Ulam's type stability," Abstract and Applied Analysis, vol. 2012, Article ID 716936, 41 pages, 2012.

[34] Y. L. Fang, X. You, and Z. X. Chen, "New phase fitted and amplification fitted Numerov-type methods for periodic IVPs with two frequencies," Abstract and Applied Analysis, vol. 2012, Article ID 742585, 15 pages, 2012. 


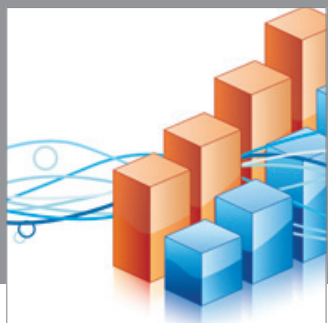

Advances in

Operations Research

mansans

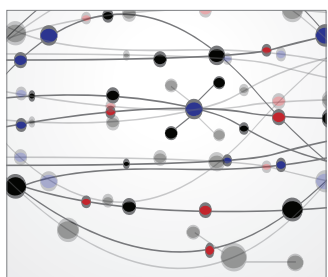

The Scientific World Journal
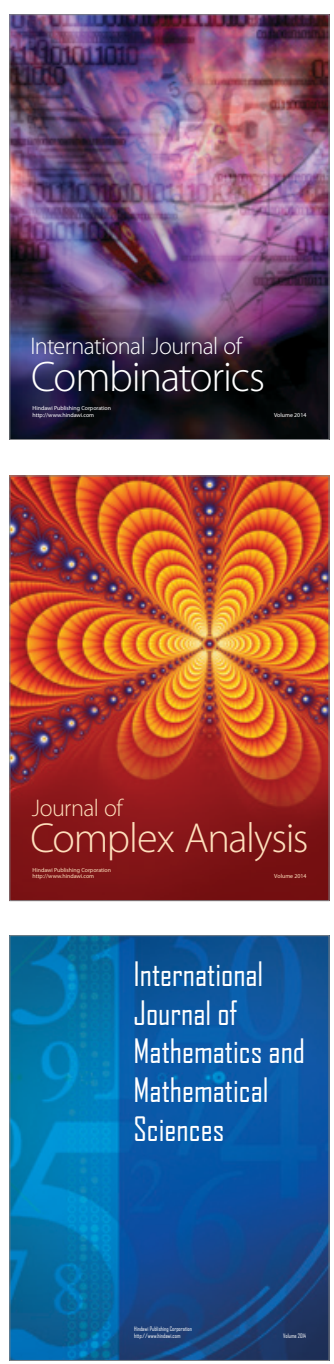
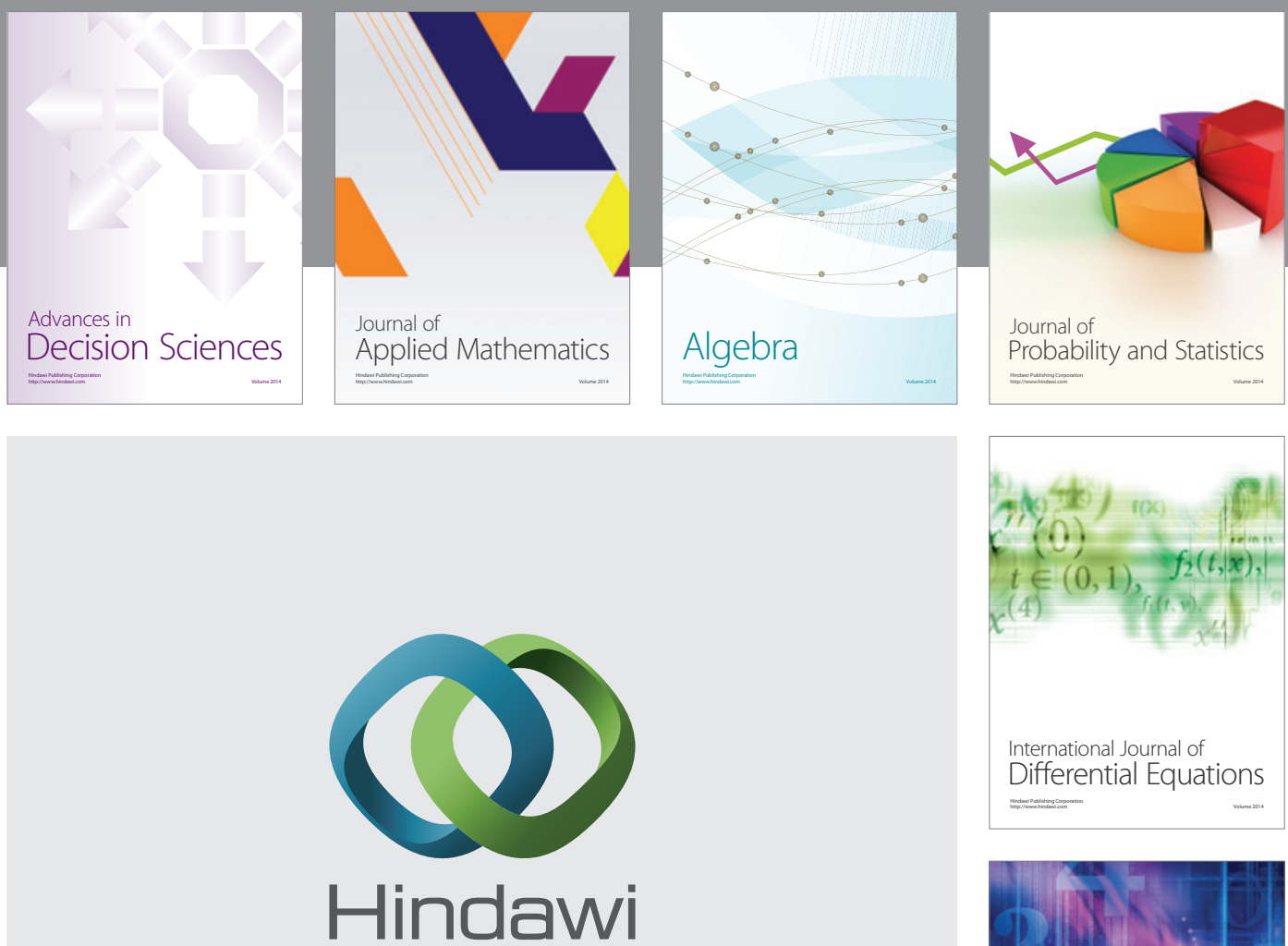

Submit your manuscripts at http://www.hindawi.com
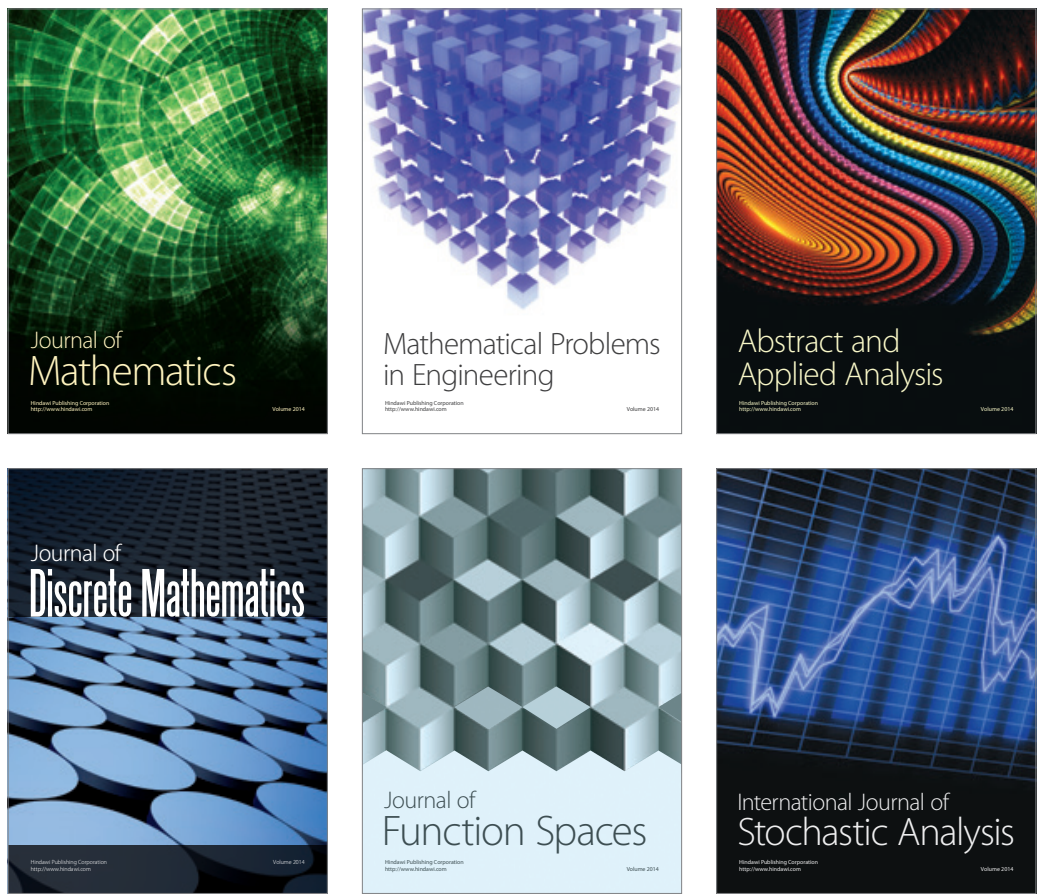

Journal of

Function Spaces

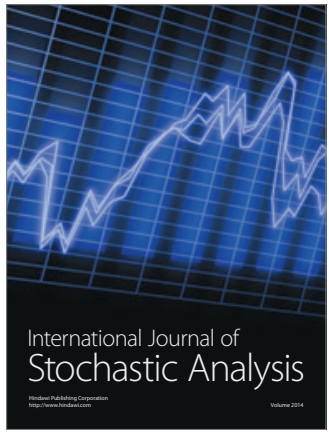

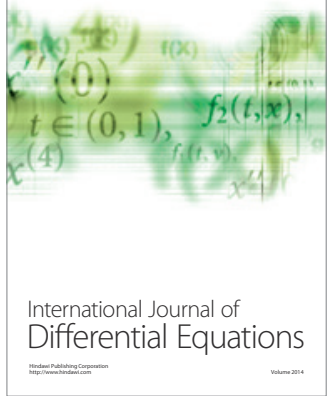
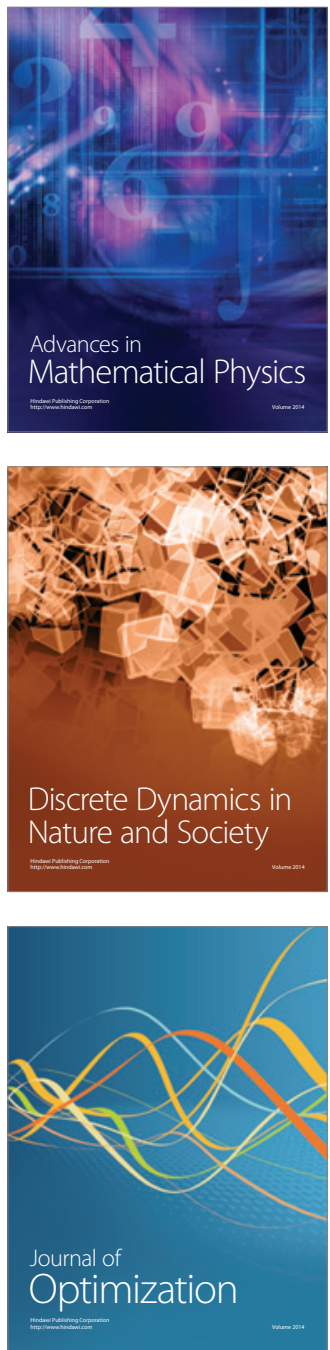